\title{
Salt Effects on the Structure and Dynamics of Interfacial Water on Calcite Probed by Equilibrium Molecular Dynamics Simulations
}

\author{
Azeezat Ali, Tran Thi Bao Le and Alberto Striolo* \\ Department of Chemical Engineering, University College London, London WC1E 6BT \\ United Kingdom \\ David R. Cole \\ School of Earth Sciences, The Ohio State University, Columbus, Ohio 43210 \\ United States of America
}

\section{Supporting Information}
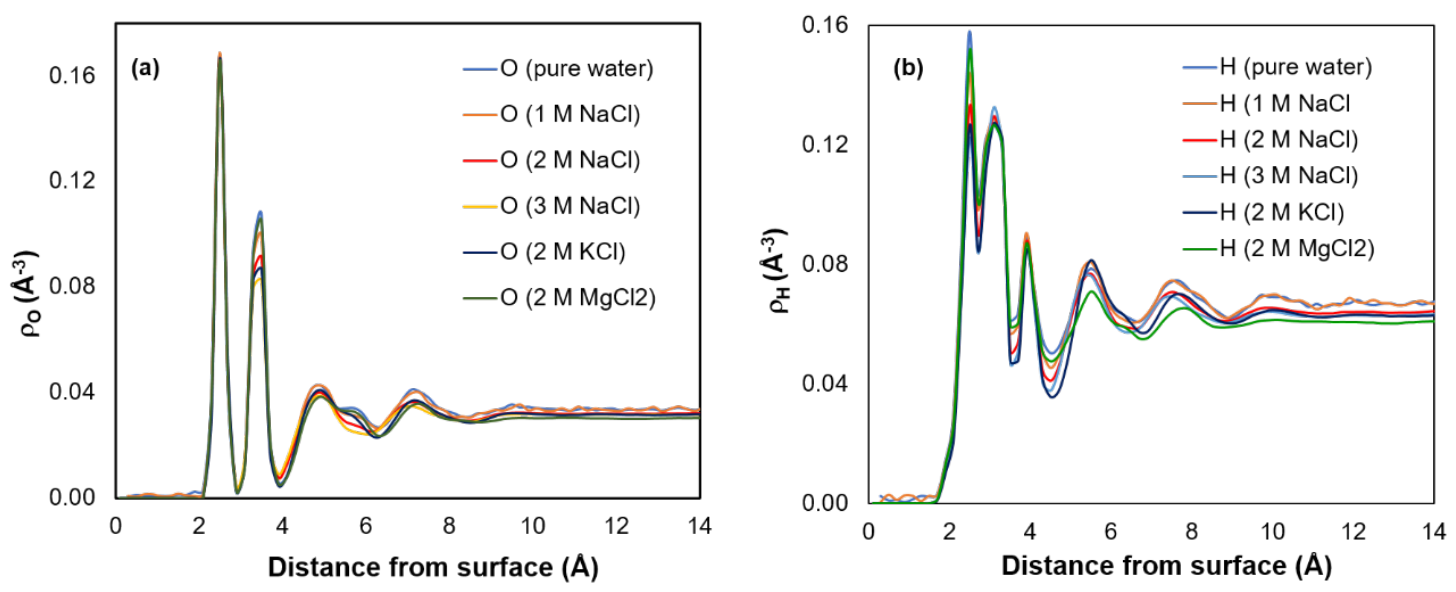

Figure S1. Atomic density profiles along the $\mathrm{z}$ direction, vertical from the surface, for (a) oxygen atoms and (b) hydrogen atoms for all systems considered in this work. The reference (i.e., $z=0$ ) is defined by the z-position of the plane of the top calcium atoms on the calcite surface. These simulations were conducted using the force field proposed by Xiao et al. ${ }^{35}$ to describe calcite and they were conducted at $298 \mathrm{~K}$. 


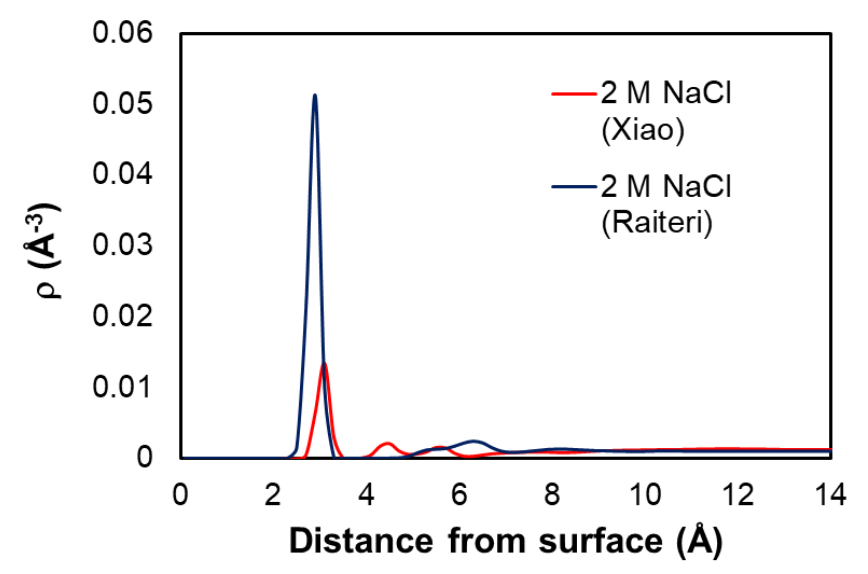

Figure S2. Atomic density profiles along the $\mathrm{z}$ direction, vertical from the surface, for $\mathrm{Na}^{+}$ions in $2 \mathrm{M} \mathrm{NaCl}$ systems. The reference (i.e., $\mathrm{z}=0$ ) is defined by the $\mathrm{z}$-position of the plane of the top calcium atoms on the calcite surface. These simulations were conducted using the force field proposed by Xiao et al. ${ }^{35}$ (red curve) and Raiteri et al. ${ }^{36}$ (blue curve) to describe calcite and they were conducted at $298 \mathrm{~K}$.
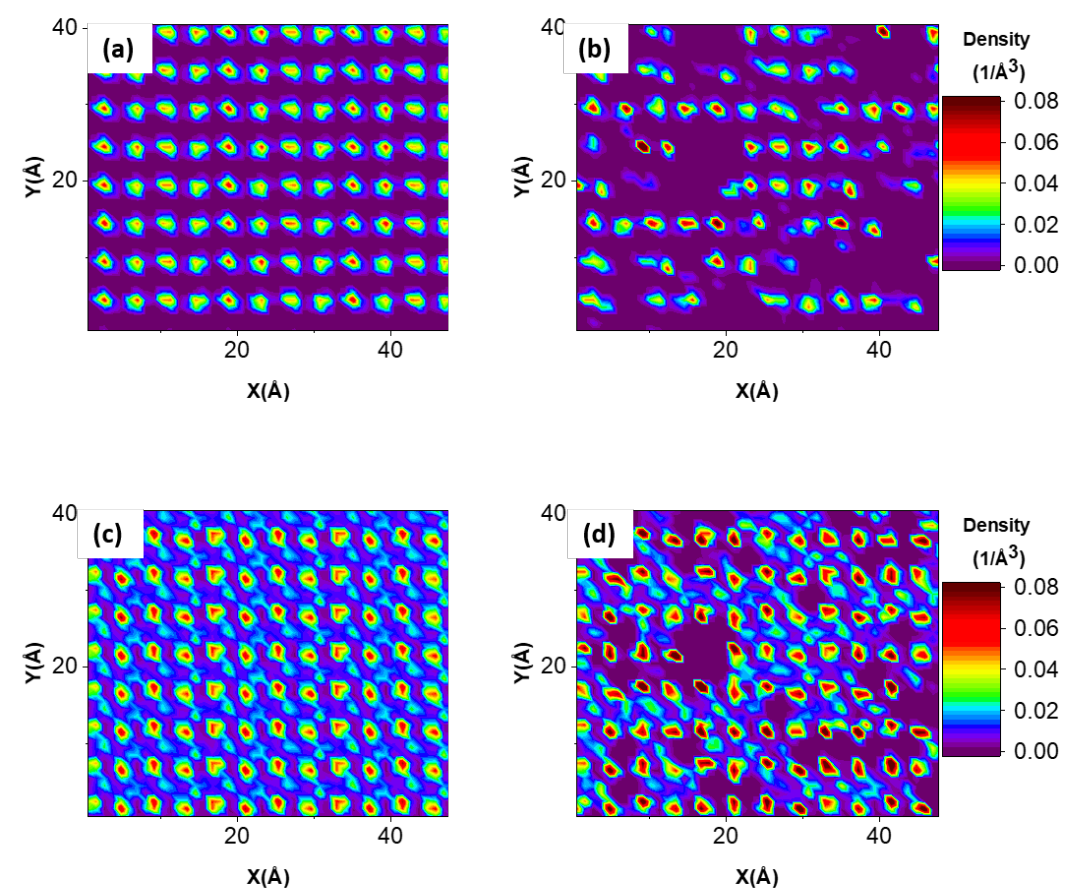

Figure S3. Surface density distributions of water oxygen atoms (panels (a) and (b)) and water hydrogen atoms (panels (c) and (d)) in the second hydration layer for systems with no salt (panels (a) and (c)) and $2 \mathrm{M} \mathrm{NaCl}$ (panels (b) and (d)). These simulations were conducted using the force field proposed by Raiteri et al., ${ }^{36}$ applying the LJ parameters from Shen et al. ${ }^{37}$ to describe calcite. The colour bar expresses density in the units of $1 / \AA^{3}$. 

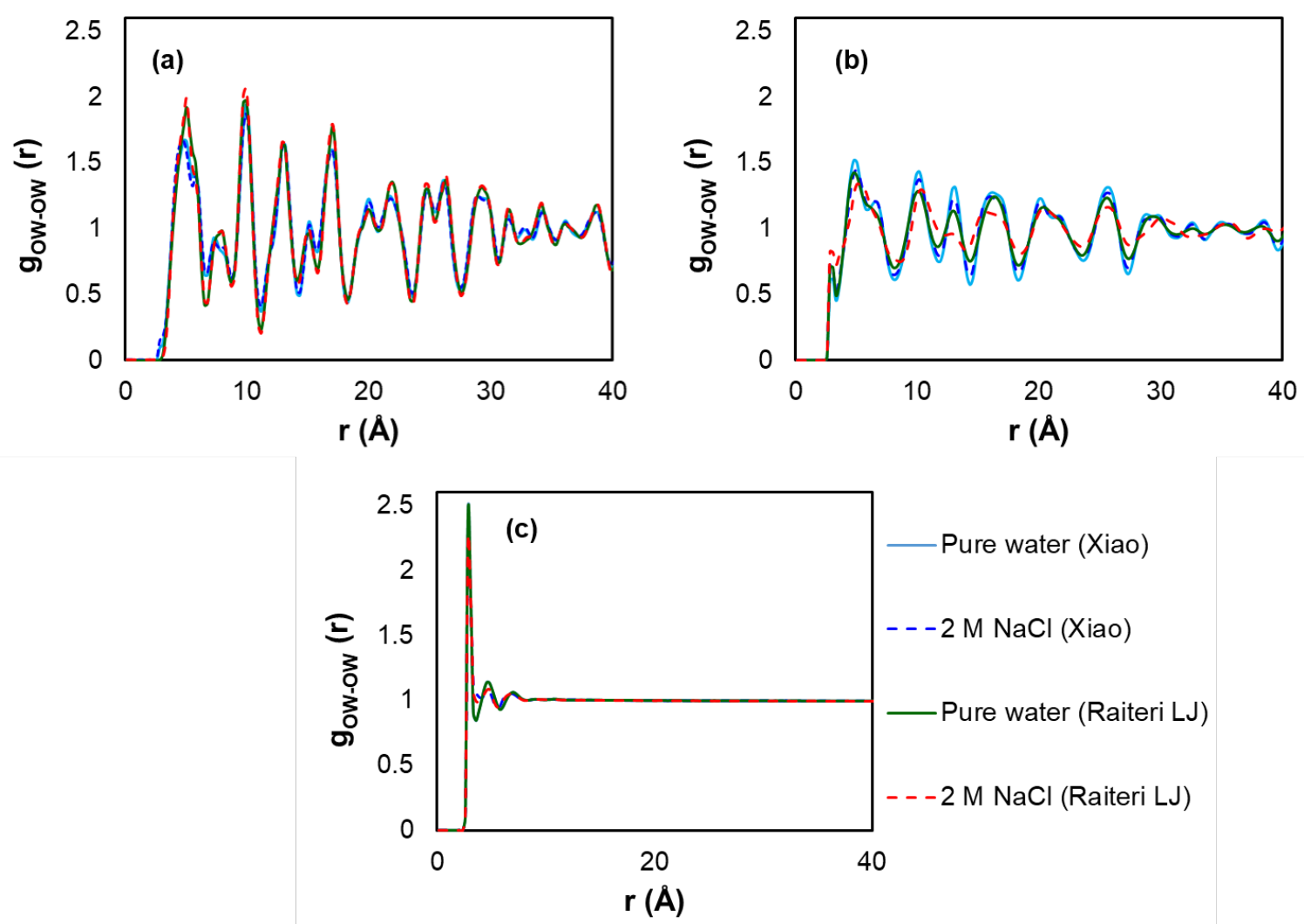

Figure S4. Oxygen-oxygen radial distribution functions for water molecules in the first and second hydration layers, as well as in the bulk (>13 $\AA$ ) for systems with pure water and $2 \mathrm{M}$ $\mathrm{NaCl}$. These simulations were conducted using the force field proposed by Xiao et al. ${ }^{35}$ and Raiteri et al., ${ }^{36}$ applying the LJ parameters from Shen et al. ${ }^{37}$ to describe calcite. The simulated temperature was $298 \mathrm{~K}$. 


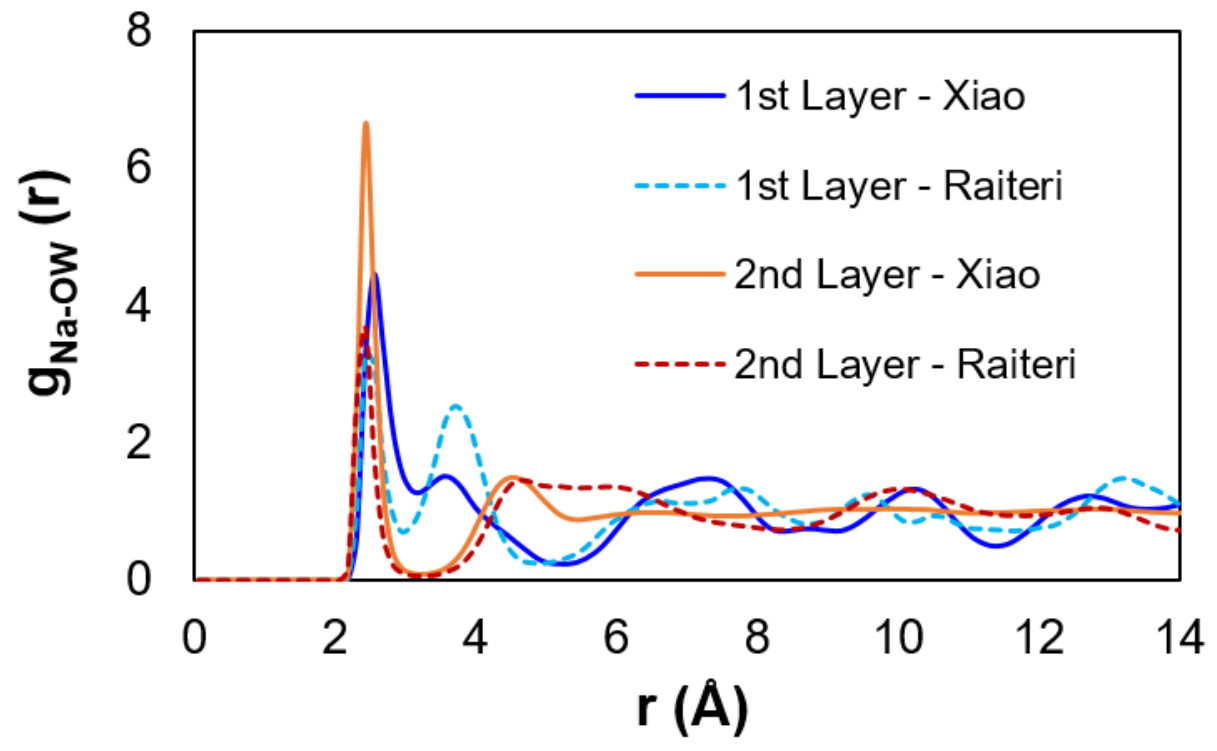

Figure S5. Radial distribution functions between water oxygen atoms and $\mathrm{Na}^{+}$in the first and second hydration layers for systems with $2 \mathrm{M} \mathrm{NaCl}$. These simulations were conducted using the force field proposed by Xiao et al. ${ }^{35}$ and Raiteri et al., ${ }^{36}$ applying the LJ parameters from Shen et al. ${ }^{37}$ to describe calcite. The simulations were conducted at $298 \mathrm{~K}$. 